\title{
Exploration of pregnancy blues among first time expectant fathers
}

\begin{abstract}
Context: First time expectant fathers need mental health resources just as much as new expectant mothers as paternal prenatal mental health has an impact on maternal and child outcome.
\end{abstract}

Aim: To explore the prevalence of pregnancy blues among first time expectant fathers.

Settings and design: Purposive sampling was used to recruit first time expectant fathers during their low risk partners at 4- 37 weeks of gestation visit to antenatal clinics at MCH services.

Materials \& methods: With informed consent the first time expectant fathers were screened for the prevalence of pregnancy blues by Pregnancy Blues Questionnaire consisting of 15 Likert items, scored on a 4-point scale, ranging from mild blue (1) to intense blue (4), with the scores ranging from 15 to 60. SPSS version 20 used for statistical analysis.

Results: The prevalence of meaningful pregnancy blues among first time expectant fathers during partners pregnancy was $89.79 \%$ (cut of score $>30$ ). The men's' blues were primarily related to ambivalent feelings about pregnancy, health status of their partner and unborn baby, overwhelming sense of responsibilities, changes in their physical and mental well-being, life style changes , psychological and social isolation form partner, family and friends, labor process, poor support form health personnel, their parenting capacity, balance between work and family life.

Conclusion: Health professionals are in an ideal position to provide support to first time expectant fathers. Offering the first time expectant fathers the space to talk about how they are feeling and including them in the prenatal care is the first step to minimize pregnancy blues among men.

Keywords: first time expectant fathers, pregnancy blues, prenatal period
Volume 2 Issue 5 - 2017

\author{
Samia Saud Al Furaikh,' Ganapathy \\ Thilagavathy \\ 'Consultant Pediatric Gastroenterologist, King Saud Bin Abdul- \\ Aziz University for Health Sciences, Saudi Arabia \\ ${ }^{2}$ Department of Nursing, King Saud Bin Abdul-Aziz University \\ for Health Sciences, Saudi Arabia
}

Correspondence: Ganapathy Thilagavathy, Department of Nursing, King Saud Bin Abdul-Aziz University for Health Sciences, Saudi Arabia,Tel 966I 3562 9000,

Email thilkg@gmail.com

Received: March 15, 2017| Published: May 09, 2017
Key message: A paradigm shift is required in $\mathrm{MCH}$ services, from a focus on women to a broader family perspective with the focus firmly on men friendly initiatives to promote healthier concepts of fatherhood within families.

\section{Introduction}

\section{"Unexpressed emotions will never die. They are buried alive and will come forth later in uglier ways."- Sigmund Freud}

The mental health of men during their partner's pregnancy remains a neglected area of research and one that is not adequately addressed during the transition to prospective fatherhood in maternal child health care. The prenatal period for first time expectant fathers has been considered as a time of crisis brought about by emotional, psychological and social stress. ${ }^{1}$ This could be because men undergo psychological reorganization during this time and aiming to adhere to a father image based on close involvement with the family, child care- an image for which they never inherited a role model never. ${ }^{1,2}$ For many men prenatal period can be a time of heightened risk for mental health and emotional responses. ${ }^{3}$ During this period, men gradually realize the lifestyle changes they are facing and however, the fact that they cannot experience the physical aspect of pregnancy allows them to delay the identity transformation process. ${ }^{1-4}$ The main difficulties they encounter during this time are linked to diminished independence. Moreover, they are forced by the events to learn a new approach to life, so as to deal with a new sense of feeling powerlessof not having control over the situation and to accept that they are entering a new phase of their lives. ${ }^{1,3-5}$ The conflicting mental states produced by these components are frequently expressed through moodiness, irritability ${ }^{3,4}$ anxiety frustration and a negative perception of self., ${ }^{4,5}$

The current fatherhood culture has begun redefining the father role once viewed exclusively in terms of breadwinner and disciplinarian, to one of fathers also being involved in the care of children of all ages. ${ }^{3,5}$ The transition for the woman to motherhood is more clearly structured and sign posted than for man to fatherhood and support services are structured around the needs of woman rather those of man. ${ }^{3-5}$ Genesoni who investigated men's psychological transition to fatherhood from pregnancy of the partner suggest that men may feel they actually need help and support from their partner during pregnancy ${ }^{1}$ to successfully negotiate the changes in their life style and relationship in the move towards fatherhood. Their friends, relatives and health professionals may unwittingly increase their sense of isolation, feelings of loss or helplessness when they focus on the mother. ${ }^{4-7}$

Some men also report experiencing ambivalent feelings in pregnancy and have differing views from their partner's about 
the couple relationship, attending to each person's needs and role of father may foster anxiety and difficulty in the acceptance of the transition. ${ }^{5-8}$ Focusing on the kind of father they want to be and the role they will take on with their child is associated with bonding with the unborn infant ${ }^{6,7}$ some expectant fathers also feel they are not given sufficient information by health professionals about a range of issues around pregnancy, childbirth and postnatal period. ${ }^{6-8}$ Thus , although the majority of expectant fathers may be delighted with their partner's pregnancy and the preparations involved in becoming a parent $t^{4,6}$ it can be a challenging time with stresses arising from the transition to changing their roles, changes in the relationship with their partners, economic changes because of woman's maternity leave. ${ }^{2,7}$ Pregnancy is associated with an increased risk of psychological ill- health among men, especially as their partner who might usually be providing them with support, is looking for greater support from them. ${ }^{7}$ How successfully men manage this transitional period often depends on the coping strategies they employ. ${ }^{8,9}$

In the course of generating a new life, every parent will undergo some transformation of self, with the attendant emotional and psychological journey. ${ }^{3}$ For fathers, this transition has been described as a journey in limbo, through no-man's land. ${ }^{8}$ Their path is not clearly marked by body changes, medical observation, or social recognition of pregnancy, and many fathers continue to feel that the transition to fatherhood is difficult and isolating. ${ }^{7,8}$ However, a smooth transition to fatherhood is essential to children's well-being and father-child relationships. ${ }^{9}$ Research reports that a new father's experience of the antenatal period is more stressful than the postnatal period $^{8}$ Mood disorders among fathers have consequences for their offspring. A father's depression alone can also negatively affect infant development. ${ }^{9,10}$ Most people know that depression and anxiety can affect women during pregnancy and early parenthood, but men can also be at risk. ${ }^{4} \mathrm{Up}$ to 1 in 20 new expectant dads can experience prenatal depression..$^{7-10}$ Developmentally, becoming a father requires a man to build on the experiences he has had throughout childhood and adolescence. ${ }^{9,10}$

Men's psychological issues associated with fatherhood include unresolved anxieties related to their sexual maturity and development, role conflict and the competing demands of relationships, social expectations, and the financial burden associated with parenthood. ${ }^{10,11}$ Study on first-time fathers, identified anxiety, ambivalence, and difficulties in adjustment; confusion about changes in the relationship with their partner; and a need to understand their role in the pregnancy as men's key concerns that were usually unaddressed by health care professionals. ${ }^{8-12}$ The mental health of men remains a neglected area of research and one that is not adequately addressed during the transition to fatherhood. ${ }^{11}$ These findings, add weight to the argument that men should be monitored for anxiety and depression during pregnancy and men should never occupy a blind spot in reproductive health services. Thus, the purpose of this article was to explore the experiences of the first time expectant fathers during their partner's pregnancy to reinforce the importance of relevant antenatal preparation that meets effectively the needs not only of expectant women but also of expectant men.

\section{Materials and methods}

As there is a paucity of information on pregnancy blues among men, this present study examined the first-time expectant father's blues during their partners' prenatal period to provide maternal- child health care professionals with greater insight on emotional investment or feelings of expectant fathers during their partner's pregnancy, thereby informing the care and support of men during prenatal period.

An exploratory approach was undertaken to examine the firsttime expectant fathers pregnancy blues during their partners prenatal period from October 2015 to September 2016 and one hundred seven first time expectant fathers $(\mathrm{n}=107)$ were recruited when their partners were approximately 4-37 weeks pregnant, attending antenatal clinics at a larger private maternal child health care organizations, BangaloreSouth, Karnataka, India. Purposive sampling was undertaken to include men from diversified culture with a different needs and views. The inclusion criteria were the first time expectant fathers whose partners were normal low risk primigravidae with single fetus, no history of chronic diseases, infertility, medical disorders and history of psychiatrist visit. Exclusion criteria were first time expectant fathers with second marriage, age above 35 years, unplanned and unwanted pregnancy and having suffered recent stressful events. No problems were encountered while recruiting men to participate in the study. Indeed they were pleased to discuss their feelings and experiences despite their hectic schedule and although nine of them could not complete the interview due to personal work.

\section{Measurements}

To estimate the prevalence of pregnancy blues among the first time expectant fathers during their partners' prenatal period-- Pregnancy Blues Question naire was developed by the researcher. Pertinent domains were identified through literature studies, discussions with experienced midwives and obstetricians and an interview with Primiparous women, their partners and family members. Identified areas of Pregnancy Blues Questionnaire included blues related to experiencing conflicting and ambivalent emotions during pregnancy, blues related to the health/life of the partner and the unborn baby, blues related to overwhelming responsibilities on impending fatherhood, abilities to fulfill them, blues related to changes in their physical and mental well-being, psychological isolation form partner's pregnancy, social isolation from friends, family members, impending fatherhood life style and managing it, blues related to adjustment difficulties in the personal, behavioral, lifestyle and relational changes, blues related to negative attitude, and poor support from the health personnel, blues related to labor and delivery process, impending paternal parenting capabilities, financial issues, work and family balancing capabilities in paternal postpartal period.

The tool contained Pregnancy Blues Questionnaire with 15 items. The questions were presented as statements with a participant's response rate on a four -point Likert scale ranging from mild blue (1), moderate blue (2), severe blue (3) and intense/extreme level of blue (4) and the scores ranged from 15 to 60 . The maximum scores were 60 and the cut-off score was set priori at and above 30 to evaluate the prevalence of meaningful pregnancy blues. Scores above 30 indicated pregnancy blues among the first time expectant fathers ranging from severe to extreme/intense level. Scores below 15 were considered mild level of blue, a score from 15 to 30 equates to moderate level of pregnancy blues. Content validity of the tool had been established by the experts and the content validity index was -CVI-0.85. Pilot study was conducted among 12 first time expectant men and the reliability of this questionnaire yielded 0.89 with Cronbach's alpha.

The researcher interviewed each first time expectant father during their prenatal visit. Details about their age, employment type, 
antenatal care, sources of support and information were collected. The semi-structured interviews were carried out using a Pregnancy Blues Questionnaire to gain an understanding on feelings and experiences of first time expectant fathers during their partner's prenatal period. The interview was carried out in flexible manner and the direction in which the men wanted to take it. Interviews were manually documented and anonymity of the data was ensured by allocating numbers.

\section{Ethical approval}

This study follows the ethical regulations and conforms to the Declaration of Helsinki. Ethical approval was obtained from the hospital ethical committee. The participants were given both oral and written information about the study, invited to ask questions and to discuss their participation with family or care provider's . The researcher explained to the participants that their participation would be voluntary. After the information, written informed consent was obtained from all participants.

Participants were assured that they could excuse themselves or withdraw from the study whenever they wanted, without giving any explanation, at any time during the study with no consequences on their partners care. They were guaranteed that findings would not be linked to individuals and that all study events and materials would maintain confidentiality. They were also informed that they were free not to answer any question that they felt not comfortable to answer. Participants were not considered to be at risk of harm. The first time expectant fathers were informed that the duration of the interview would be approximately 30-40 minutes.

\section{Statistical analysis}

Statistical analysis was conducted using SPSS 20 (Chicago, III USA). Descriptive statistics -frequencies, mean scores and mean percentage were performed on all items and non-parametric Chisquare/Fishers exact probability tests were used to find the association of pregnancy experiences with sociodemographic variables. An alpha of less than 0.05 was considered significant in all tests.

\section{Results}

A total of $n=107$ first time expectant fathers were approached to participate in the study, $\mathrm{n}=3(2.8 \%) 8.4$ refused to participate and $\mathrm{n}=9(8.4 \%)$ withdrew from the study due to their busy schedule of work after answering three to seven questions and were excluded from the study. Overall, $n=95(88.8 \%)$ first time expectant fathers were interviewed during their partners' 4 to 37 weeks of gestation.

\section{Participants' characteristics}

The participants were between 23 to 34 years old, with an average age of 25.7 years $( \pm 6.59)$, and the mean gestational age of their partner was 28.3 weeks $( \pm 8.1)$. With regard to educational status majority of them $97.9 \%$ were graduates and post graduates and the rest $2.1 \%$ had their technical and allied health education. All of them were employed and from middle and upper middle socioeconomic background.

\section{The prevalence of meaningful pregnancy blues}

The Pregnancy Blues scores ranged from 18 to 57 (out of possible scores of 60) among the first time expectant fathers. The prevalence of meaningful pregnancy blues among first time expectant fathers during partners pregnancy was $89.79 \%$ (cut of score $>30$ ) and the mean score was $54.35(\mathrm{SD}=9.621)$. Majority of the first time expectant fathers $n=74(77.89 \%)$ had pregnancy blues varying from severe to intense level (cut of score $>30$ ). A very few had a mild pregnancy blues $\mathrm{n}=5(5.26 \%$; scores $<15)$, a few $\mathrm{n}=16$ scored in the moderate range (16.84\%; scores 15-30). The vast majority of men $n=49(66.22 \%)$ reported intense/extreme level of pregnancy blues (scores $>45$ ) Table 1.

Table I Levels of pregnancy blues among first time expectant fathers.

\begin{tabular}{ll}
\hline Levels of pregnancy blues & First time expectant father's F (\%) \\
\hline Mild & $05(5.26 \%)$ \\
Moderate & $16(16.84 \%)$ \\
Severe & $25(26.32 \%)$ \\
Intense/extreme & $49(57.58 \%)$ \\
\hline
\end{tabular}

$\mathrm{N}=95$

\section{Pregnancy blues among first time expectant fathers}

Although the first time expectant fathers felt happy about partner's' pregnancy, the most vivid and commonly expressed pregnancy blues, was related to experiencing ambivalent, conflicting and diverse emotions during prenatal period. All of the participants felt unprepared for becoming fathers. They articulated feelings of ignorance, anxiety, guilty, worry, scared and unknown fear about impending fatherhood. Majority of the participants (94.5\%) reported that they experienced a spectrum of emotions varying from joy, happiness, excitement, pride, out of control, sense of achievement, surprise to shocked, guilty, apprehension, frightened, disappointment, fear and sadness during prenatal period.

Prenatally, although all the first time expectant fathers felt excited about upcoming life with a baby they also expressed feelings of apprehension about the consequences of the pregnancy, health status of their partner and unborn baby. Most men (92.3\%) expressed health related anxieties and worries about the partner and their unborn baby: partners morning sickness, sleep, rest, work, pain, weight gain, discomfort, moods, food intake, rest and baby's movement, kicking, growth, health and anomalies. Regarding the responsibilities majority of expectant fathers $(89.5 \%)$ were scared and worried about the overwhelming sense of responsibilities that were looming over them and felt insecure about their new roles, expectations as father, all of a sudden in their care free life.

The impact of the pregnancy on men's wellbeing revealed that highest proportion of the participants experienced mental $(82.3 \%)$ and physical changes $(50.75 \%)$ during their partners' pregnancy. Men made explicit reference to experiencing psychological changes, affecting their mental health, in response to their partner's pregnancy. They voiced concerns over their emotional/mood changes from feeling euphoric to depressive states, emotionally drained, frustrated, exhausted, irritable, sleep disturbances coupled with bad dreams and night mares. Participants mentioned that their physical healths were affected during pregnancy. Some expectant fathers reported loss of appetite, Gastrointestinal disturbances, changes in body weightweight loss \& gain, generalized body pain, abdominal pain, difficulty in breathing and cramps in lower limbs which they attributed to partners' pregnancy.

Psychological isolation from the partner's pregnancy changes were expressed by $75 \%$ of the expectant fathers. They reported that they 
feel emotionally heavy with mixed feelings, conflicting emotions for unknown reasons which they were unable to reveal to their partners and a felt a strange sense of emotional detachment from their partners. About $74.25 \%$ participants narrated that the pregnancy imposed restrictions on their social gatherings, meeting their friends, play, party and enjoyment out side and resulted in social isolation. Some men $(65 \%)$ pointed that the prenatal period caused some undesirable changes of transitioning to fatherhood for which they were not yet ready.

Many men $(62.8 \%)$ reported that they need vast amount of support, explanations, clarifications, discussions and positive concern with respect for them from the health care professionals. They also expressed that the health professionals should be open to acknowledge and value their feelings and emotions that they were going through during their partner's pregnancy without labeling them as emotionally incompetent to handle changes in their life during the prenatal period. Pregnancy blues experienced by fathers regarding fear about labor and delivery process was the next reason for many men blues in pregnancy. Some men expressed that $(62.3 \%)$ were worried about their partner's capabilities to tolerate labor pain, operative procedures, course of labor and baby's conditions and health care personnel attitudes.

Many men felt insecure and even ambivalent about their parenting capacity $(59.7 \%)$, balancing work and family life $(58.05 \%)$ and financial issues $(60.25 \%)$ and these were other common factors for some expectant fathers' prenatal blues. Some men they expressed that pregnancy posed a significant adjustment difficulties for them in their physical, psychological, behavioral and social life styles in preparation for impending fatherhood. There was insignificant association of first time expectant fathers pre baby blues symptoms with their sociodemographic variables at 0.05 level of significance. Overall $(89.79 \%)$ had experienced meaningful pregnancy blues varying from severe to intensive level during their partners' prenatal period Table 2

Table 2 Prevalence of pregnancy blues among first time expectant fathers

\begin{tabular}{|c|c|c|c|}
\hline S. No & Pregnancy blues among first time expectant fathers & Mean score & Mean \% \\
\hline I. & Blues related to experiencing ambivalent and conflicting emotions during partner's pregnancy. & 3.78 & 94.5 \\
\hline 2. & Blues related to the health status of their partner and unborn baby. & 3.69 & 92.3 \\
\hline 3. & Blues related to overwhelming sense of responsibilities. & 3.58 & 89.5 \\
\hline 4. & Blues related to their ability to fulfill new roles and expectations. & 3.49 & 87.3 \\
\hline 6. & Blues related to their physical well being. & 2.03 & 50.75 \\
\hline 7. & Blues related to their psychological isolation From pregnant partner. & 3 & 75 \\
\hline 8. & Blues related to their Social isolation from friends, family members & 2.97 & 74.25 \\
\hline 9. & Blues related to their impending fatherhood lifestyle and managing it. & 2.6 & 65 \\
\hline 10. & Blues related to their adjustment difficulties in the personal, lifestyle and relational changes. & 2.58 & 64.5 \\
\hline II. & Blues related to the negative attitudes/inadequate Support from health professionals. & 2.51 & 62.8 \\
\hline 12. & Blues related to labor \& delivery process. & 2.49 & 62.3 \\
\hline 13. & Blues related to financial issues. & 2.41 & 60.25 \\
\hline 14. & Blues related to impending paternal parenting role. & 2.39 & 59.75 \\
\hline 15. & Blues related to work and family balancing life in the prospective postpartal paternal period. & 2.34 & 58.5 \\
\hline
\end{tabular}

$\mathrm{N}=95$

\section{Discussion}

This study explores the prevalence of pregnancy blues among first time expectant fathers, from culturally diverse backgrounds. It highlights the salient issues which they encountered during their transition to impending fatherhood, which may be of importance in enhancing reproductive health professionals' knowledge and understanding in this area.

\section{Prevalence of pregnancy blues among first time expectant fathers}

It is often assumed that the physical and psychological effects of pregnancy are only experienced by women, a concept that is reflected in the bulk of literature addressing this theme. However, pregnancy also affects male partners in ways that relate to their physiological and psychological constitution, a concept of potential importance in reproductive analyses. ${ }^{8-13}$ The prevalence of pregnancy blues among first time expectant fathers in the present study (89.79\%)) indicates that common life transition, such as partner's pregnancy may be stressful and a major life change for new expectant fathers. Men found this transition difficult, reported experiencing pregnancy blues accompanied by changes in mental wellbeing. Paulson reported similar findings that the prenatal period impose greater risk of deterioration in mental health among men than the postpartal period and their parenting capacity can be compromised by deterioration in mental health with significant variation from anxiety to depression. ${ }^{7,11}$

\section{Participants characteristics}

The lack of association between first time expectant fathers pregnancy blues and socio demographic variables in the current study 
analyzed by Chi- square/Fisher's Exact Probability test may represent the averaged impact of expectant fatherhood across the various positives and negatives when all new fathers to be are considered together. Within antenatal care it is the primacy of the woman's health which is deemed paramount often at the expense of the man's., 3,11 Providing new expectant fathers with timely, relevant information about prenatal period physical and psychological changes may reduce paternal pregnancy blues and anxiety and increase their coping skills. Improved antenatal education to meet the needs of fathers and early awareness and intervention may limit the negative impact of pregnancy blues on parenting attitudes and behavior. ${ }^{12-14}$

\section{Pregnancy blues among first time expectant fathers}

In the present study most of the participants reported that they experienced a range of diverse and conflicting emotions during their partner's pregnancy and their emotional responses spanned the entire spectrum of emotions from overwhelming joy, out of control happiness, fear, scared, guilty, terrified, worry, to disappointment. All expectant fathers, expressed some form of anxiety/worry as a consequence of the pregnancy; the most importantly the health status of their partner and unborn baby. They were worried about the partner's health such as morning sickness, food intake, sleep, and rest, variation in moods, her feelings, baby's movement, kicking, growth, congenital anomalies, impairment and a whole well-being of their unborn baby. Similar findings were reported by researchers that experiencing anxiety, worry, internal conflict, guilt, uncertainty, and mixed emotions appear to constitute the normal emotional and psychological repertoire for men during the early stages of pregnancy. ${ }^{8,14} \mathrm{All}$ of the expectant fathers expressed concerns about the overwhelming sense of responsibility they felt looming over them, and their ability to fulfill new roles and expectations. They were very apprehensive about giving up their present life to prospective, responsible paternal life loaded with unexpected and huge responsibilities on their shoulders all of a sudden in their life. Research reported that anxiety and worry are common psychological processes for expectant fathers during their partner's pregnancy, with many men worrying about the health status of their partner and unborn baby and how they will adapt to and fulfill the expectations and responsibilities of their new role. ${ }^{13,14}$ It seems that experiencing anxiety and worries during their partner's pregnancy form part of the normal experiential trajectory for expectant fathers and is not unduly mediated by prior parenting experiences. ${ }^{7,12}$

For many men prenatal period can be a time of heightened risk for mental health and emotional responses. During this period, men gradually realize the lifestyle changes they are facing and however, the fact that they cannot experience the physical aspect of pregnancy allows them to delay the identity transformation process. ${ }^{12,14}$ In the current study changes in physical and mental wellbeing were a significant aspect of some first time expectant fathers' experience of pregnancy blues. Participants made explicit reference to experiencing physical changes such as nausea, vomiting, giddiness, loss of appetite and constipation which they attributed to their partner's pregnancy. Regarding mental wellbeing participants mentioned that their emotional/mood states were affected during the pregnancy. Some expectant fathers acknowledged changes in their mental health status -becoming moody, irritable, feeling angry, sad for unknown reasons experiencing anxiety, frustration, exhaustion and a negative perception of self. It is widely accepted that men can experience psychological changes, affecting their mental health, in response to their partner's pregnancy. ${ }^{8,12}$ It has also been documented that some men experience psycho-physiological changes during pregnancy known as the
Couvade syndrome. The development of physical and mental changes in a man is an unconscious attempt to feel more involved and a part of the pregnancy process. ${ }^{12,14}$

It is important to note that many participants expressed feeling emotionally isolated from the pregnancy and they felt sense of emotional and physical withdrawal from the partner's pregnancy experiences which may be related to contemporary male hegemony and cohort beliefs. This psychological response is consistent with how men think and feel during the moratorium phase of pregnancy. ${ }^{14-16}$ Which is characterized by them often feeling removed and emotionally distanced from the pregnancy experience because they do not have first-hand experience of their partners' pregnant body. ${ }^{10,15,16}$

Despite many men wanting to be involved during the antenatal care, expectant fathers in this study commented on feeling unsupported, excluded and marginalized, by health care professionals' attitude. This highlights the fact that health professionals may not be aware of expectant fathers' needs during pregnancy. The sense of separation and detachment that many men already experience during pregnancy seems to be propagated by health services not making them feel valued and acknowledged in the antenatal period. Draper ${ }^{3}$ and Finnbogadottir ${ }^{13}$ reported consistent findings in their studies that men spoke about experiencing negative attitudes from health professionals which propagated their sense of alienation. This finding is surprising considering the socio-political shift regarding fathers' role in modern family systems and the well-established empirical evidence highlighting the benefits of their involvement in mother and child wellbeing. 5,8

Many men commented on the personal, lifestyle, relational changes and adjustments they started to make in response to impending fatherhood such as reducing the frequency of intake of alcohol, smoking and going out with friends. Few men lamented on the social adjustments they experienced like drifting away from friend's gatherings, and social chatting. Although the vast majority of participants were positive about impending fatherhood roles, there were difficulties expressed relating to changes to sense of self and integrating changes in their existing life structure. Finnbogadottir ${ }^{13}$ also found that expectant fathers were reticent when it came to sacrificing personal interests in preparation for fatherhood. These findings tentatively suggest that men find it difficult to let go or change familiar lifestyle activities during the period of transition. Striking balance between the work and demands at home was the next pregnancy blues for the expectant fathers as many of them were worried about upcoming financial issues. A lack of male roles, suggested for men to emulate may be a reason why some men find difficulty in adjusting to the new role, responsibilities, and lifestyle that fatherhood brings. ${ }^{16,17}$

Men's experiences of support during pregnancy juxtaposed with the expectations placed upon them by society, highlight a disparity between the evidence base and clinical practice in maternity services; ${ }^{4,10}$ it seems the scientific evidence is not significantly impacting on how maternity services are delivered to take account of the role, expectations, and experiences of expectant fathers. ${ }^{1,17}$ Expectant fathers' experiences of antenatal support appear to capture the prevailing systemic attitude which influences how antenatal/maternity services are provided. This dominant attitude has highlighted what some authors have termed mother-centrism in today's parenting culture. ${ }^{17-19}$ This term alludes to the bias which exists in parenting programs, support groups, and attitudes of health service providers, which are set up to meet the needs of women, 
marginalize those of men $^{8,14}$ and perpetuate men's ambivalence about their role in raising children and acknowledging their own needs. ${ }^{10,16}$ It is of vital importance for health professionals who work with couples in the antenatal period to acknowledge that men tend to view their role during pregnancy as providers, and not receivers of support. ${ }^{8,13,14}$ Although the men in the current study acknowledged a lack of maleoriented support in the antenatal period, some also voiced a reluctance to seek help. It may be this lack of help-seeking behavior by men which may convey the impression, to health professionals, that they are coping, when they may not be.

Health professionals should show an interest in expectant fathers by acknowledging the role they have to play in the pregnancy process, and by asking how they are coping as the pregnancy progresses, and the types of support they would find useful. ${ }^{16,19}$ Findings from studies $^{10-18}$ reported that expectant fathers mentioned the value of one-to-one support; this suggests that men will not honestly open up in front of other men or indeed their partners. Dedicated one-toone support interventions have obvious cost implications attached to them, but health professionals could, whenever appropriate, offer the expectant father some time to speak to them alone during their routine clinical practice, It has been suggested that if fathers continue to feel unsupported and undervalued, it may lead to reduced family involvement, psychological impairments, and a diminished ability to effectively parent. ${ }^{1,3,7,16-18}$ It is important for health professionals to spend time assessing the mental health of fathers, as the presence of paternal depression is positively linked to maternal depression in the prenatal period. ${ }^{15-19}$

The needs of men and women during pregnancy are inter-related and are not worlds apart. It is however, important to acknowledge differences. ${ }^{12-14}$ Expectant fathers often feel anxious, conflicted, dissatisfied, and the societal pressure to feel and behave in a certain way, during pregnancy. ${ }^{3,8,9}$ Health professionals should support expectant fathers, and help them to feel more a part of and involved in the pregnancy process, as involvement seems to significantly influence overall psychological adjustment. ${ }^{3,5,15-18}$

\section{Implications for practice}

Men are generally reluctant to seek advice regarding emotional health issues. ${ }^{3,8}$ Economic and cultural factors may make it impossible for new expectant fathers to accompany their partners to every health service visit. However, more could be done to provide for a father's need for support when he does attend with his partner, and specific information on their role as expectant fathers could be directed to men during the prenatal period. ${ }^{1,3,17,18}$ Regular contacts with mothers during the perinatal period through antenatal clinics, $\mathrm{MCH}$ practitioners have an opportunity to supply information for their partners on parenting from a father's perspective. This will assist new fathers to distinguish between mood disorders and the normal stress occasioned by a prenatal period, and to make them aware of existing support services.

\section{Limitation}

No claims are made as to the wider generalizability of the findings of this study, as the men taking part were purposively recruited, and were from a variety of different cultural backgrounds and geographical areas across the Karnataka state.

\section{Conclusion}

It is accepted that women, who are not adequately supported emotionally and practically during pregnancy, are at higher risk of developing complications during pregnancy, and psychological problems in the post-natal period. ${ }^{19}$ As well as the cost in human suffering this has a significant financial cost to healthcare providers as well. It stands to reason that if expectant fathers are better supported themselves, they will be in a stronger position to give their partners the support they need, the best way for health professionals to achieve this is to first acknowledge that the needs of men and women are similar, and try and make expectant fathers feel valued by encouraging their involvement throughout pregnancy. ${ }^{10-18}$ Reproductive health care ignores the father's mental well-being and is focused on the mother ${ }^{19}$ and child during the pregnancy and childbirth and this has a huge impact on the family as whole. ${ }^{4-11}$ This study has highlighted that prenatal period is a more stressful period for most men and they should be monitored for pregnancy blues during their partners' antenatal period. Since men are less likely to seek professional help for any emotional health problems, a proactive approach towards targeting the wellbeing of expectant fathers may be beneficial. With an awareness of the psychological tasks facing new fathers, a health professional is able to take opportunities to assess and foster progress in a man's preparation for the role of father, with significant long term benefit for the man, his partner and his child.

\section{Acknowledgements}

None.

\section{Conflict of interest}

The author declares no conflict of interest.

\section{References}

1. Genesoni L, Tallandini MA. Men's psychological transition to fatherhood: an analysis of the literature, 1989-2008. Birth. 2008;36(4):305-318.

2. Henwood K, Procter J. The 'good father': reading men's accounts of paternal involvement during the transition to first-time fatherhood. $\mathrm{Br} J$ Soc Psychol. 2003;42(Pt 3):337-355.

3. Draper J. Men's passage to fatherhood: an analysis of the contemporary relevance of transition theory. Nurs Inq. 2003;10(1):66-77.

4. Golian Tehrani S, Bazzazian S, et al. Pregnancy Experiences of FirstTime Fathers in Iran: A Qualitative Interview Study. Iranian Red Crescent Medical Journal. 2015;17(2):e12271.

5. Deave T, Johnson D. The transition to parenthood: what does it mean for fathers. J Adv Nurs. 2008;63(6):626-633.

6. Draper. Blurring, moving and broken boundaries: men's encounters with the pregnant body. Sociol Health Illn. 2003;25(7):743-767.

7. Condon JT, Boyce P, Corkindale CJ. The First-Time Fathers Study: a prospective study of the mental health and wellbeing of men during the transition to parenthood. Aust N Z J Psychiatry. 2004;38(1-2):56-64.

8. Currid TJ. Psychological issues surrounding paternal perinatal mental health. Nurs Times. 2005;101(5):40-42.

9. Constance MD. Interactions between adolescent fathers and health care professionals during pregnancy, Labor, and Early Postpartum. JOGNN. 2009;38(3):290-299.

10. Paulson JF, Bazemore SD. Prenatal and postpartum depression in fathers and its association with maternal depression: a meta-analysis. JAMA. 2010;303(19):1961-1969.

11. Boyce P, Condon J, Barton J, et al. First-Time Fathers' Study: psychological distress in expectant fathers during pregnancy. Aust $N Z J$ Psychiatry. 2007;41(9):718-725. 
12. Kazmierczak M, Kielbratowska B, Pastwa-Wojciechowska B, et al. Couvade Syndrome among Polish expectant fathers. Medical Science Monitor. International Medical Journal of Experimental and Clinical Research. 2013;19:132-138.

13. Finnbogad ottir H, Svalenius EC, Persson EK. Expectant first-time fathers' experiences of pregnancy. Midwifery. 2003;19(2):96-105.

14. Kaye DK, Kakaire O, Nakimuli A, et al. Male involvement during pregnancy and childbirth: men's perceptions, practices and experiences during the care for women who developed childbirth complications in Mulago Hospital, Uganda. BMC Pregnancy Childbirth. 2014;14:54 p.

15. Hamilton K, Kavanagh D, Connolly J, et al. Baby steps - An online program promoting the well-being of new mothers and fathers: A study protocol. JMIR Research Protocols. 2016;5(3):e140.
16. Jeffery T, Luo KY, Kueh B, et al. Australian Fathers' Study: What Influences paternal engagement with antenatal care. The Journal of Perinatal Education. 2015;24(3):181-187.

17. Fagerskiold A. A change in life as experienced by first-time fathers. Scand J Caring Sci. 2008;22(1):64-71.

18. Fletcher R, Silberberg S, Christopher GM. Addressing depression and anxiety among new fathers. Med J Aust. 2006;185:461-463.

19. Darvill R, Skirton H, Farrand P. Psychological factors that impact on women's experiences of the first-time motherhood: a qualitative study of the transition. Midwifery. 2010;26(3):357-366. 